\title{
Composição química, atividade antibacteriana in vitro e toxicidade em Artemia salina do óleo essencial das inflorescências de Ocimum gratissimum L., Lamiaceae
}

\author{
Lenise L. Silva, ${ }^{1}$ Clarissa G. Heldwein, ${ }^{1}$ Luiz G. B. Reetz, ${ }^{2}$ Rosmari Hörner, ${ }^{2}$ \\ Carlos A. Mallmann, Berta M. Heinzmann ${ }^{*}, 1$
}

${ }^{1}$ Departamento de Farmácia Industrial, Centro de Ciências da Saúde, Universidade Federal de Santa Maria, Prédio

26, Campus Universitário, 97105-900 Santa Maria-RS, Brasil,

${ }^{2}$ Departamento de Análises Clínicas e Toxicológicas, Centro de Ciências da Saúde, Universidade Federal de

Santa Maria, Prédio 26, Campus Universitário, 97105-900 Santa Maria-RS, Brasil,

${ }^{3}$ Departamento de Medicina Veterinária Preventiva, Centro de Ciências Rurais, Universidade Federal de Santa

Maria, Prédio 44, Campus Universitário, 97105-900 Santa Maria-RS, Brasil.

\begin{abstract}
RESUMO: O óleo essencial das inflorescências de Ocimum gratissimum L., Lamiaceae foi obtido por hidrodestilação e analisado por CG/EM. Os constituintes majoritários identificados foram eugenol $(81,94 \%)$ e $\gamma$-muuroleno (12,58\%). O óleo essencial das inflorescências demonstrou atividade antibacteriana frente a todas as cepas bacterianas testadas pelo método de microdiluição em caldo. Merece destaque a atividade verificada frente às cepas resistentes de Enterococcus faecalis, Staphylococcus aureus e Escherichia coli. Os valores obtidos de concentração inibitória mínima (CIM) e a concentração bactericida mínima (CBM) variaram, respectivamente, entre 0,5-2 $\mathrm{mg} / \mathrm{mL}$ e $1-4 \mathrm{mg} / \mathrm{mL}$. Valores de CL50 de 233,8 (200,7-272,0) $\mu \mathrm{g} / \mathrm{mL}$ para o óleo essencial e 186,1 $(144,1-228,5) \mu \mathrm{g} / \mathrm{mL}$ para o eugenol, utilizado como controle positivo, foram observados frente à Artemia salina L.
\end{abstract}

Unitermos: Ocimum gratissimum, inflorescências, óleo essencial, atividade antibacteriana.

\begin{abstract}
Chemical composition, antibacterial activity in vitro and brine-shrimp toxicity of the essential oil from inflorescences of Ocimum gratissimum L., Lamiaceae." The essential oil obtained by hydrodistillation of the inflorescences of Ocimum gratissimum L. was analyzed by GC/MS. The main constituents were eugenol (81.94\%) and $\gamma$-muurolene (12.58\%). Antibacterial activity was shown against all assayed strains by the broth microdilution method. It's worth noting the activity against resistant strains of Enterococcus faecalis, Staphylococcus aureus and Escherichia coli. Minimum inhibitory concentration (MIC) and minimum bactericidal concentration (MBC) values ranged between $0.5-2 \mathrm{mg} / \mathrm{mL}$ and $1-4 \mathrm{mg} / \mathrm{mL}$, respectively. Preliminary toxicity assayed by the brine-shrimp (Artemia salina L.) test showed LC50 values of $233.8(200.7-272.0) \mu \mathrm{g} / \mathrm{mL}$ and 186.1 (144.1 - 228.5) $\mu \mathrm{g} / \mathrm{mL}$, respectively for the essential oil and eugenol (positive control).
\end{abstract}

Keywords: Ocimum gratissimum, inflorescences, essential oil, antibacterial activity.

\section{INTRODUÇÃO}

Vários estudos têm sido realizados com produtos do metabolismo vegetal secundário, visando encontrar substâncias com atividade antimicrobiana que possam servir como alternativas terapêuticas efetivas contra as infecções por microrganismos resistentes a antibióticos (Acosta et al., 2003). Uma das maiores fontes de pesquisa nessa área é a confirmação da atividade de plantas utilizadas popularmente com esse fim. Ocimum gratissimum L., Lamiaceae, conhecida popularmente como alfavaca, é utilizada no Brasil como anti-séptico local contra fungos e bactérias (Matos, 1994; Morais et al., 2005; Silva et al., 2006). Devido a esta atividade, é uma das espécies consideradas para inclusão no programa de fitoterapia do Sistema Único de Saúde (SUS). Suas inflorescências em particular são utilizadas para o tratamento de problemas digestivos, flatulência, gripe, tosse, prurido, estresse, dor de cabeça, fadiga e como sedativo e expectorante (Alcântara Júnior et al., 2005; Albuquerque et al., 2007).

As partes aéreas de $O$. gratissimum fornecem óleo essencial, que pode ser classificado em três quimiotipos (eugenol, timol e geraniol) de acordo com seus constituintes principais (Vieira et al., 2001). A literatura relata para esta 
espécie as atividades antinociceptiva (Rabelo et al., 2003), antioxidante (Trevisan et al., 2006) e antimicrobiana (Jedličková et al., 1992; Nguefack et al., 2004). Contudo, não existem trabalhos relacionando estudos de atividade biológica à composição química do óleo essencial obtido exclusivamente das inflorescências de $O$. gratissimum.

A avaliação de toxicidade é indispensável para considerar um tratamento seguro (Cáceres, 1996). O teste de letalidade com Artemia salina L. é um método simples na pesquisa de produtos naturais (Meyer et al., 1982), que possui uma boa correlação com testes de toxicidade aguda oral in vivo (Parra et al., 2001). O procedimento determina valores de concentração letal média (CL50), em $\mu \mathrm{g} / \mathrm{mL}$, de compostos e extratos, sendo que inúmeras substâncias ativas conhecidas apresentam toxicidade por este teste (Meyer et al., 1982).

$\mathrm{O}$ presente trabalho relata a atividade antibacteriana do óleo essencial obtido das inflorescências de $O$. gratissimum frente a cepas bacterianas catalogadas e isolados clínicos, visando determinar a real contribuição do óleo das inflorescências para a atividade antimicrobiana, anteriormente relatada para as folhas desta espécie. Adicionalmente, são também descritas sua toxicidade para Artemia salina L, bem como uma possível correlação destas atividades com sua composição química.

\section{MATERIAL E MÉTODOS}

\section{Material vegetal}

As inflorescências de Ocimum gratissimum L., Lamiaceae, foram coletadas em dezembro de 2006 no Campus da Universidade Federal de Santa Maria, Santa Maria, RS. Material testemunha, identificado por Adelino Alvares Filho, encontra-se depositado no herbário do Departamento de Biologia da UFSM sob o registro $\mathrm{n}^{\circ}$ SMDB 11167.

\section{Obtenção do óleo essencial e identificação dos constituintes}

A extração do óleo essencial das inflorescências $(224,3 \mathrm{~g})$ de $O$. gratissimum foi realizada pelo método de hidrodestilação por $3 \mathrm{~h}$, utilizando o aparelho de Clevenger modificado (Farmacopéia Brasileira, 1988). O óleo essencial foi armazenado em frasco de vidro âmbar vedado e conservado à $-20^{\circ} \mathrm{C}$ até o momento das análises.

As análises por cromatografia gasosa acoplada à espectrometria de massas (CG/EM), visando à identificação e quantificação dos componentes do óleo essencial, foram realizadas em um sistema hifenado AGILENT 6890, equipado com um detector seletivo de massas série 5973. Parâmetros de análise: split inlet 1:100; gás carreador: $\mathrm{He}(1 \mathrm{~mL} / \mathrm{min})$; coluna capilar de sílica fundida HP5MS (Hewlett Packard, $5 \%$ fenilmetilsiloxano, $30 \mathrm{~m}$ x 0,25 $\mathrm{mm}$, espessura do filme: $0,25 \mu \mathrm{m})$; programa de análise:
$40{ }^{\circ} \mathrm{C}$ (Ti) por $4 \mathrm{~min}, 40-260^{\circ} \mathrm{C}, 4^{\circ} \mathrm{C} / \mathrm{min}$; temperatura do injetor: $220^{\circ} \mathrm{C}$; temperatura da interface: $250{ }^{\circ} \mathrm{C}$; energia de ionização: $70 \mathrm{eV}$; banco de dados: NIST, 1998.

Os componentes foram identificados com base no índice de retenção (IR), determinados através da utilização de uma curva de calibração de uma série homóloga de $n$-alcanos $\left(\mathrm{C}_{8}-\mathrm{C}_{32}\right)$ injetados nas mesmas condições cromatográficas da amostra e nos modelos de fragmentação dos espectros de massas, sendo ambos comparados com dados da literatura (Adams, 2001).

A concentração dos constituintes foi calculada através da área integral de seus respectivos picos, relacionada com a área total de todos os constituintes da amostra, obtida pela análise em sistema de cromatografia em fase gasosa.

\section{Avaliação da atividade antibacteriana do óleo essencial}

A atividade antibacteriana do óleo essencial foi avaliada utilizando a metodologia de microdiluição em caldo, com base no documento M7-A6 (NCCLS, 2003), conforme descrito por Murari et al. (2008).

As cepas bacterianas foram armazenadas a $-20{ }^{\circ} \mathrm{C}$ no banco de microrganismos do Laboratório de Bacteriologia (LAB) do DACT, UFSM, RS, Brasil. Foram utilizadas nos testes cepas bacterianas catalogadas e isoladas em culturas de diferentes espécimes clínicos como sangue, fezes, entre outros, obtidas no Hospital Universitário de Santa Maria (Tabela 2).

O óleo essencial foi dissolvido em etanol 95\%, de forma a obter uma solução estoque com concentração de $160 \mathrm{mg} / \mathrm{mL}$. Diluições em série foram realizadas de forma a obter concentrações entre 8-0,0156 mg/mL. Ampicilina foi utilizada como antibiótico padrão, em concentrações entre $10-0,00976 \mathrm{mg} / \mathrm{mL}, 2-0,00195 \mathrm{mg} / \mathrm{mL}$ e $0,1-$ $0,0000976 \mathrm{mg} / \mathrm{mL}$, determinadas de acordo com o perfil de resistência microbiana. Controles negativo, positivo e do produto foram realizados para cada bactéria testada. Todos os testes foram efetuados em triplicata.

A concentração inibitória mínima (CIM) é definida como a menor concentração capaz de inibir completamente o crescimento microbiano, nas cavidades de microdiluição, conforme detectado a olho nu, enquanto que a concentração bactericida mínima (CBM) é a menor concentração em que o composto é capaz de agir como bactericida.

\section{Teste de toxicidade para Artemia salina L.}

O teste de toxicidade com náuplios de Artemia salina (Leach) foi realizado de acordo com metodologia adaptada de Vanhaecke et al. (1981). Os ovos de A. salina $(30 \mathrm{mg})$ foram eclodidos entre $22-29^{\circ} \mathrm{C}$ em salina artificial (23 g/L de sal marinho e $0,7 \mathrm{~g} / \mathrm{L}$ de bicarbonato de sódio em água destilada). Após 24 h, dez náuplios de Artemia 
salina foram transferidos para placas de Petri contendo 9 $\mathrm{mL}$ de salina artificial e $1 \mathrm{~mL}$ da amostra a ser testada. Os testes foram realizados em triplicata, em duas repetições. A contagem dos náuplios mortos e vivos foi realizada após $24 \mathrm{~h}$.

O óleo essencial (103 mg) foi dissolvido em etanol $(1 \mathrm{~mL})$ e diluído em salina artificial de forma a obter concentrações finais de 515, 257,5, 103 e 10,3 $\mu \mathrm{g} /$ $\mathrm{mL}$. Eugenol foi utilizado como controle positivo, em concentrações finais de 514, 257, 135,1 e 13,5 $\mu \mathrm{g} / \mathrm{mL}$. Controle negativo foi realizado com etanol diluído 1:100 em salina artificial. Os valores de CL50 e seus intervalos de confiança de $95 \%$ foram calculados por análise de Probitos (Tabela 2).

\section{RESULTADOS E DISCUSSÃO}

As inflorescências de $O$. gratissimum forneceram $0,6 \%(\mathrm{~m} / \mathrm{m})$ de óleo essencial contendo cinco constituintes
(Tabela 1) identificados através da análise por CG/EM. O composto majoritário, identificado como eugenol $(81,94 \%)$, determina o quimiotipo do material vegetal estudado.

Rendimentos e composição química similares aos obtidos neste estudo foram verificados por Vieira et al. (2001) para o óleo essencial obtido das partes aéreas de $O$. gratissimum. Contudo em relação ao óleo obtido apenas das inflorescências desta espécie, Kothari et al. (2005) relataram para plantas cultivadas em clima tropical semi-árido valores de rendimento $(0,4 \%)$, teor de eugenol $(55,8 \%)$ e de $\beta$-cariofileno $(1,4 \%)$ inferiores aos observados neste trabalho. Estas discrepâncias possivelmente estejam relacionados a variações de diferentes fatores ambientais aos quais a espécie vegetal foi submetida, como luz, temperatura e umidade. A alteração destes fatores tem demonstrado influenciar significativamente a emissão de compostos voláteis e o rendimento e composição de óleos essenciais (Dudareva et al., 2004).

Tabela 1. Composição química do óleo essencial das inflorescências de Ocimum gratissimum L., Lamiaceae.

\begin{tabular}{|c|c|c|c|c|c|}
\hline Componente & Estrutura molecular & $\%$ & $\operatorname{Tr}(\min )$ & IRcal & IRref \\
\hline Eugenol & & 81,94 & 26,895 & 1359 & 1359 \\
\hline$\alpha$-Copaeno & $\mathrm{H}$ & 1,57 & 27,491 & 1377 & 1377 \\
\hline$\beta$-Cariofileno & & 3,14 & 28,902 & 1421 & 1419 \\
\hline$\gamma$-Muuroleno & & 12,58 & 30,831 & 1483 & 1480 \\
\hline$\delta$-Cadineno & & 0,77 & 32,098 & 1525 & 1523 \\
\hline
\end{tabular}

\% = Porcentagem relativa; $\mathrm{Tr}=$ Tempo de retenção; IRcal = Índice de retenção calculado; IRref = Índice de retenção de referência 
Dentre os sesquiterpenos identificados constatase a relativa predominância de compostos com esqueleto cadinano $(\gamma$-muuroleno, $\alpha$-copaeno e $\delta$-cadineno $)$. A origem biossintética comum destes compostos a partir do cátion germacradienílico ou $(E, Z)$-germacrílico pode ser uma possível explicação para este fato (Steele et al., 1998; Bülow \& König, 2000).

Monoterpenos não foram detectados no óleo essencial das inflorescências de $O$. gratissimum. A ausência destes constituintes pode estar relacionada ao metabolismo vegetal e/ou dano mecânico à planta (Figueiredo et al., 2007). Para plantas dos gêneros Ocimum sp. e Perilla sp., a síntese de fenilpropanóides, especialmente eugenol e compostos relacionados, é baseada em efeitos dominantes, enquanto que a produção de monoterpenos corresponde a um fator recessivo (Sangwan et al., 2001).

$O$ óleo essencial das inflorescências de $O$. gratissimum apresentou valores de CIM entre $0,5-2 \mathrm{mg} /$ $\mathrm{mL}$ para as bactérias Gram-positivas testadas e $1-2 \mathrm{mg} / \mathrm{mL}$ para as Gram-negativas (Tabela 2). A maior resistência das bactérias Gram-negativas à ação de óleos essenciais já é descrita na literatura como decorrente da grande complexidade da dupla membrana apresentada por estes microrganismos, a qual limita a difusão de compostos hidrofóbicos, através de sua cobertura lipopolissacarídica (Burt, 2004; Holley\& Patel, 2005).

O mecanismo da ação antibacteriana dos compostos fenólicos não está totalmente elucidado. Alguns estudos indicam que estes exerceriam seus efeitos na membrana através de numerosos mecanismos, como ruptura, complexação de substrato, inativação enzimática e quelação de metais. Em relação aos demais componentes do óleo essencial em estudo, a atividade antibacteriana pode ser conferida pela lipofilia dos hidrocarbonetos de estrutura terpênica, que permite sua partição nos lípidios da membrana celular, aumentando sua permeabilidade e desestabilizando a estrutura da célula bacteriana (Sikkema et al., 1995; Cowan, 1999).

A alteração doperfildeácidos graxos erompimento da membrana bacteriana, com liberação de constituintes intracelulares, foram verificados frente a microrganismos Gram-positivos e Gram-negativos para o eugenol (Walsh et al., 2003; Gill \& Holley, 2006a; Di Pasqua et al., 2007). Adicionalmente, a inibição da atividade da ATPase ligada à membrana de $E$. coli e L. monocytogenes também foi demonstrada experimentalmente e relacionada à ação bactericida deste composto (Gill \& Holley, 2006a, b).

Em relação aos constituintes de natureza terpênica, apenas $\beta$-cariofileno e $\delta$-cadineno têm atividade antimicrobiana descrita frente a fungos e bactérias pelo método de microdiluição em caldo (Skaltsaa et al., 2003). Estes dados, no entanto, mostram-se controversos, uma vez que não foi detectada atividade para $\beta$-cariofileno frente a E. coli enteropatogênica (EPEC) e E. coli enterotoxigênica pela mesma metodologia (Duarte et al., 2007). Possíveis explicações seriam as diferenças existentes entre as cepas testadas e a baixa solubilidade dos hidrocarbonetos sesquiterpênicos em água a qual, segundo Griffin et al. (1999), estaria relacionada a sua baixa atividade antimicrobiana.

Para o óleo essencial das folhas de $O$. gratissimum são considerados possíveis mecanismos de atividade antimicrobiana a permeabilização da membrana citoplasmática verificada em Listeria innocua (Nguefack et al., 2004) e efeitos anti-virulência em Shigella sp. por diminuição na atividade da protease extracelular, conteúdo de ramnose do o-lipopolissacarídeo e incidência de invasividade (Iwalokun et al., 2003). Estes mecanismos,

Tabela 2. Atividade antibacteriana do óleo essencial (OE) das inflorescências de Ocimum gratissimum L., Lamiaceae.

\begin{tabular}{|c|c|c|c|c|}
\hline \multirow[t]{2}{*}{ Microrganismo testado } & \multicolumn{2}{|c|}{$\mathrm{CIM}(\mathrm{mg} / \mathrm{mL})$} & \multicolumn{2}{|c|}{$\mathrm{CBM}(\mathrm{mg} / \mathrm{mL})$} \\
\hline & $\mathrm{OE}$ & Ampicilina & $\mathrm{OE}$ & Ampicilina \\
\hline \multicolumn{5}{|l|}{ Gram-positivo } \\
\hline Bacillus cereus ATCC 14579 & 0,5 & $0,78 \cdot 10^{-3}$ & 1 & $31,25.10^{-3}$ \\
\hline Bacillus cereus & 1 & $15,6 \cdot 10^{-3}$ & 4 & 0,5 \\
\hline Enterococcus faecalis ATCC 51299 & 2 & $3,12 \cdot 10^{-3}$ & 2 & $>0,05$ \\
\hline Staphylococcus aureus ATCC 25923 & 1 & $0,39.10^{-3}$ & 4 & $>0,05$ \\
\hline $\mathrm{MRSA}^{\mathrm{a}}$ & 2 & $31,25 \cdot 10^{-3}$ & 4 & $>1$ \\
\hline \multicolumn{5}{|l|}{ Gram-negativo } \\
\hline Escherichia coli $\mathrm{ESBL}^{\mathrm{b}}$ & 2 & $>5$ & 2 & $>5$ \\
\hline Escherichia coli EPEC $^{c}$ & 2 & $3,9 \cdot 10^{-3}$ & 2 & 0,25 \\
\hline Shigella sp. & 1 & $3,9 \cdot 10^{-3}$ & 2 & 0,5 \\
\hline Shigella flexneri & 2 & $3,9 \cdot 10^{-3}$ & 2 & $>1$ \\
\hline Salmonella sp. & 1 & $3,9 \cdot 10^{-3}$ & 2 & $>1$ \\
\hline Salmonella choleraesuis ATCC 10708 & 1 & $25 \cdot 10^{-3}$ & 2 & 0,05 \\
\hline
\end{tabular}

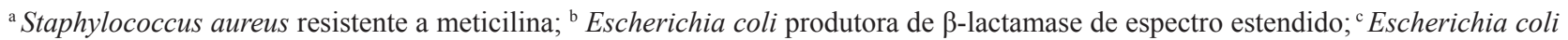
enteropatogênica. 
contudo, não podem ser extrapolados para o óleo essencial das inflorescências de $O$. gratissimum analisado neste trabalho, uma vez que os dados de composição química dos óleos essenciais, utilizados para a realização dos estudos acima citados, não estão disponíveis para comparação.

Dentre as bactérias Gram-positivas testadas, foram verificados maiores valores de CIM para os isolados clínicos de $B$. cereus, causador de endocardite bacteriana, e $S$. aureus resistente a meticilina, em relação a suas respectivas cepas catalogadas. Estes resultados confirmam sua maior resistência e não surpreendem, uma vez que isolados clínicos freqüentemente mostram-se resistentes inclusive aos antimicrobianos de utilização terapêutica (Neely \& Holder, 1999). Adicionalmente, foi detectada atividade bactericida com valores idênticos de CIM e CBM (2 mg/mL) para a cepa padrão de Enterococcus faecalis resistente à vancomicina e para o isolado clínico de Escherichia coli produtora de beta-lactamase de espectro estendido, o que torna o óleo essencial testado uma possível fonte de novos antimicrobianos frente a estes microrganismos, cujas opções terapêuticas são extremamente limitadas (Bruin \& Riley, 2007).

O óleo essencial das inflorescências também demonstrou ação bactericida e, em alguns casos, bacteriostáticafrenteabactérias Gram-negativas causadoras de diarréia, como EPEC, Shigella sp. e Salmonella sp. Estes resultados fornecem uma possível explicação para o uso popular da planta inteira como antidiarréico em países africanos (Kaou et al., 2008). Contudo, os valores de CIM verificados foram superiores aos anteriormente descritos frente a isolados clínicos de EPEC para o óleo essencial obtido a partir das folhas (Duarte et al., 2007).

$\mathrm{Na}$ avaliação da toxicidade de compostos ativos e extratos vegetais para Artemia salina, um valor de CL50 inferior a $1000 \mu \mathrm{g} / \mathrm{mL}$ permite considerar o composto como tóxico (Meyer et al., 1982). No presente trabalho, tanto o óleo essencial das inflorescências [CL50=233,8 (200,7$272,0) \mu \mathrm{g} / \mathrm{mL}$ ] quanto o eugenol [CL50=186,1 (144,1$228,5) \mu \mathrm{g} / \mathrm{mL}$ ] apresentaram toxicidade sem diferenças estatísticas entre si. Estes dados toxicológicos, segundo Parra et al. (2001), podem ser correlacionados com testes de toxicidade aguda oral em animais.

Embora a atividade antibacteriana detectada seja de amplo espectro, merecendo destaque a ação frente a microrganismos resistentes e isolados clínicos locais, os altos valores de CIM e CBM obtidos quando comparados ao antibiótico padrão e a toxicidade observada para Artemia salina desaconselham o uso interno de monopreparados à base das inflorescências de $O$. gratissimum. Alternativa para sua utilização com esta finalidade seria em associações com antibióticos, fato este já descrito na literatura para o óleo essencial das folhas (Jedličková et al., 1992; Iwalokun et al., 2003), ou em preparações de uso tópico no caso de lesões infectadas, uma vez que foi verificada neste trabalho atividade frente a $S$. aureus, cepa causadora de infecções cutâneas. Entretanto, outros estudos são necessários para comprovar a viabilidade e segurança destas proposições, bem como a inocuidade toxicológica das mesmas.

\section{AGRADECIMENTOS}

À CAPES pelo apoio financeiro.

\section{REFERÊNCIAS}

Acosta M, González M, Araque M, Velazco E, Khourl N, Rojas L, Usubillaga A 2003. Composición química de los aceites esenciales de Ocimum basilicum L. var basilicum, O. basilicum L. var purpurenscens, O. gratissimum L., y $O$. tenuiflorum L., y su efecto antimicrobiano sobre bacterias multirresistentes de origen nosocomial. Rev Facultad de Farmacia 45: 19-24.

Adams RP 2001. Identification of essential oil components by gas chromatography/quadrupole mass spectroscopy. Illinois: Allured Publishing Corporation.

Albuquerque UP, Medeiros PM, Almeida ALS, Monteiro JM, Lins Neto EMF, Melo JG, Santos JP 2007. Medicinal plants of the caatinga (semi-arid) vegetation of $\mathrm{NE}$ Brazil: A quantitative approach. J Ethnopharmacol 114: 325-354.

Alcântara Júnior JP, Osuna JTA, Queiroz SROD, Rios AP 2005. Ethnobotanical and ethnopharmacological surveys on medicinal plants from the municipality of Itaberaba-BA for cultivation and conservation. Sitientibus Ser Cienc Biol 5: 39-44.

Bruin MA, Riley LW 2007. Does vancomycin prescribing intervention affect vancomycin-resistant enterococcus infection and colonization in hospitals? A systematic review. BMC Infectious Diseases 7: 24.

Bülow N, König WA 2000. The role of germacrene D as a precursor in sesquiterpenes biosynthesis: investigations of acid catalyzed, photochemically and thermally induced rearrangements. Phytochemistry 55: 141-168.

Burt S 2004. Essential oils: their antibacterial properties and potential applications in foods - a review. Int $J$ Food Microbiol 94: 223-253.

Cáceres A 1996. Plantas de uso medicinal en Guatemala. Guatemala: Editorial Universitaria.

Cowan MM 1999. Plant products as antimicrobial agents. Clin Microbiol Rev 12: 564-582.

Di Pasqua R, Betts G, Hoskins N, Edwards M, Ercolini D, Mauriello G 2007. Membrane toxicity of antimicrobial compounds from essential oils. J Agric Food Chem 55: 4863-4870

Duarte MCT, Leme EE, Delarmelina C, Soares AA, Figueira GM, Sartoratto A 2007. Activity of essential oils from Brazilian medicinal plants on Escherichia coli. J Ethnopharmacol 111: 197-201.

Dudareva N, Pichersky E, Gershenzon J, 2004. Biochemistry of plant volatiles. Plant Physiol 135: 1893-1902.

Farmacopéia Brasileira 1988. 4a ed. São Paulo: Atheneu.

Figueiredo R, Rodrigues AI, Costa MC 2007. Volatile composition 
of red clover (Trifolium pratense L.) forages in Portugal: The influence of ripening stage and ensilage. Food Chem 104: 1445-1453.

Gill AO, Holley RA 2006b. Inhibition of membrane bound ATPases of Escherichia coli and Listeria monocytogenes by plant oil aromatics. Int J Food Microbiol 111: 170174.

Gill AO, Holley RA 2006a. Disruption of Escherichia coli, Listeria monocytogenes and Lactobacillus sakei cellular membranes by plant oil aromatics Int $J$ Food Microbiol 108: 1-9.

Griffin SG, Wyllie SG, Markham JL, Leach DN 1999. The role of structure and molecular properties of terpenoids in determining their antimicrobial activity. Flavour Fragr $J$ 14, 322-332.

Holley RA, Patel D 2005. Improvement in shelf-life and safety of perishable foods by plant essential oils and smoke antimicrobials. Food Microbiol 22: 273-292.

Iwalokun BA, Gbenle GO, Adewole TA, Smith SI, Akinsinde KA, Omonigbehin EO 2003. Effects of Ocimum gratissimum L. essential oil at subinhibitory concentrations on virulent and multidrug-resistant Shigella strains from Lagos, Nigéria. APMIS 111: 477-482.

Jedličková Z, Mottl O, Šerý V 1992. Antibacterial properties of the Vietnamese Cajeput oil and Ocimum oil in combination with antibacterial agents. $J$ Hyg Epidemiol Microbiol Immunol 36: 303-309.

Kaou AM, Mahiou-Leddet V, Hutter S, Ainouddine S, Hassani S, Yahaya I, Azas N, Ollivier E 2008. Antimalarial activity of crude extracts from nine African medicinal plants. $J$ Ethnopharmacol 116: 74-83.

Kothari SK, Bhattacharya AK, Singh K, Ramesh SI, Rao EVSP 2005. Pre-flowering harvesting of Ocimum gratissimum for higher essential oil and eugenol yields under semiarid tropics. J Essent Oil Res 17: 212-216.

Matos FJA 1994. Farmácias vivas. Fortaleza: Editora da Universidade Federal do Ceará.

Meyer BN, Ferrigni NR, Putnam JE, Jacobsen LB, Nichols DE, McLaughlin JL 1982. Brine shrimp: A convenient general bioassay for active plant constituents. Planta Med 45: 31-34.

Morais SM, Dantas JDP, Silva ARA, Magalhaes EF 2005. Plantas medicinais usadas pelos índios Tapebas do Ceará. Rev Bras Farmacogn 15: 169-177.

Murari AL, Carvalho FH, Heinzmann BM, Michelot TM, Hörner R, Mallmann CA 2008. Composição e atividade antibacteriana dos óleos essenciais de Senecio crassiflorus var. crassiflorus. Quim. Nova 31: 1081-1084.

NCCLS 2003. National Committee for Clinical Laboratory Standards. Methods for Dilution Antimicrobial Susceptibility Tests for bacteria that grow aerobically. $6^{\text {th }}$ ed. Approved Standard M7-A6, Wayne, P. A.

Neely AN, Holder IA 1999. Antimicrobial resistance. Burns 25: 17-24.

Nguefack J, Budde BB, Jakobsen M 2004. Five essential oils from aromatic plants of Cameroon: their antibacterial activity and ability to permeabilize the cytoplasmic membrane of Listeria innocua examined by flow cytometry. Lett Appl Microbiol 39: 395-400.

Parra AL, Yhebra RS, Sandiñas IG, Buela LI 2001. Comparative study of the assay of Artemia salina L. and the estimate of the medium letal dose (LD50 value) in mice, to determine oral acute toxicity of plant extracts. Phytomedicine 8 : 395-400.

Rabelo M, Souza EP, Soares PMG, Miranda AV, Matos FJA, Criddle DN 2003. Antinociceptive properties of the essential oil of Ocimum gratissimum L. (Labiatae) in mice. Braz J Med Biol Res 36: 521-524.

Sangwan NS, Farooqi AHA, Shabih F, Sangwan RS 2001. Regulation of essential oil production in plants. Plant Growth Regul 34: 3-21.

Sikkema J, Bont AAM, Poolman B 1995. Mechanisms of membrane toxicity of hydrocarbons. Microbiol Rev 59: 201-222.

Silva MIG, Gondim APS, Nunes IF, Sousa FCF 2006. Utilização de fitoterápicos nas unidades básicas de atenção à saúde da família no município de Maracanaú (CE). Rev Bras Farmacogn 16: 455-462.

Skaltsaa HD, Demetzos C, Lazari D, Sokovic M 2003. Essential oil analysis and antimicrobial activity of eight Stachys species from Greece. Phytochemistry 64: 743-752.

Steele CL, Crock J, Bohmann J, Croteau R 1998. Sesquiterpene synthases from Grand Fir (Abies grandis). J Biol Chem 273: 2078-2089.

Trevisan MTS, Silva MGV, Pfundstein B, Spiegelhalder B, Owen RW 2006. Characterization of the volatile pattern and antioxidant capacity of essential oils from different species of the genus Ocimum. J Agric Food Chem 54: 4378-4382

Vanhaecke P, Persoone G, Claus C, Sorgeloos P 1981. Proposal for a short-term toxicity test with Artemia nauplii. Ecotoxicol Environ Saf 5: 382-387.

Vieira RF, Grayer RJ, Paton A, Simon JE 2001. Genetic diversity of Ocimum gratissimum $\mathrm{L}$. based on volatile oil constituents, flavonoids and RAPD markers. Biochem Syst Ecol 29: 287-304.

Walsh SE, Maillard J-Y, Russell AD, Catrenich CE, Charbonneau DL, Bartolo RG 2003. Activity and mechanisms of action of selected biocidal agents on Gram-positive and -negative bacteria. J Appl Microbiol 94: 240-247. 